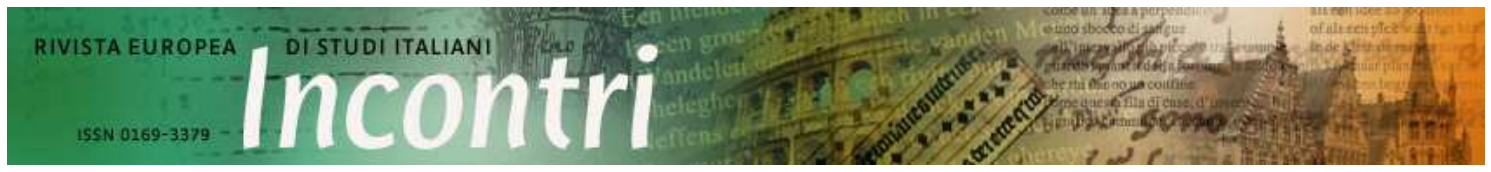

URN:NBN:NL:UI:10-1-101321 - Publisher: Igitur publishing

Content is licensed under a Creative Commons Attribution 3.0 License

Anno 26, 2011 / Fascicolo 1 - Website: www.rivista-incontri.nl

\title{
Il dibattito novecentesco sui generi tra letteratura e musica
}

\author{
Recensione di: Linda Pennings, Polemiche novecentesche, tra \\ letteratura e musica. Romanzo, melodramma, prosa d'arte, \\ Firenze, Franco Cesati Editore, 2009. ISBN: 8876673660
}

\section{Walter Geerts}

Questo è un volume per buongustai, buongustai nel campo della teoria, teoria della letteratura e teoria dell'arte, s'intende. Il titolo prepara il lettore a una materia abbastanza eterogenea. Gli stessi oggetti di studio scelti appaiono entità ibride: romanzo, melodramma, prosa d'arte. Ciò che viene offerto al lettore, tuttavia, è, sostanzialmente, una attenta disamina del crollo dell'estetica crociana nell'Italia del Novecento. Seguendo questo filo conduttore l'autrice riesce a portare a galla una serie non indifferente di ramificazioni teoriche nelle arti e tra le arti. Nessun'altra figura come Croce ha goduto, nel Novecento, di una tale supremazia nel mondo delle idee. Laddove questa fama è rimasta intatta oggi per quel che riguarda la storia, la storiografia, la filosofia, in particolare la filosofia politica, va altrettanto costatato che gli studi crociani nel campo dell'arte e dell'estetica in generale non hanno resistito all'onda contraria degli stessi artisti, delle opere e dei gusti. Nel confronto con le voci dei creatori, suoi contemporanei, il giudizio pronunciato dalla voce più autorevole del secolo spesso non ha retto. Il filosofo napoletano non seppe riconoscere il talento di Pirandello, come ignorò i futuristi. L'estetica idealistica era mal preparata per capire e penetrare l'arte che il momento storico le presentò. Chi desidera approfondire alcune tappe cruciali e vari aspetti importanti di questo processo di sfilacciamento del crocianesimo trova ampia materia di riflessione nei capitoli della Pennings.

I primi due capitoli trattano di argomenti musicali. La musica è davvero l'ospite incomodo nell'edificio crociano. Troppo legata alla tecnica e all'evoluzione delle specifiche forme, la musica non si arrende facilmente all'ortodossia crociana. Nel campo musicale la base materiale non è 'passiva' e ancor meno 'sostituibile'. Impossibile quindi fare a meno degli odiati 'generi' nel campo fortemente strutturato come la musica. Il campo letterario, d'altronde, solo in apparenza è meno immune 
da questa critica. Con grande indulgenza Briosi (p.28) nota che 'la negazione crociana dei generi letterari ha avuto il merito di porre un problema, che poi non ha risolto.' Più esplicito appare il giudizio di Torrefranca, emesso nel 1929 nei confronti della critica musicale proposta da Croce: 'Il moderno olandese volante, il critico che naviga, in perpetua tempesta, tra l'arte e la non arte...' (p.36).

Il terzo capitolo mette a confronto 'romanzo e melodramma nel sistema dei generi del primo Novecento'. L'abbinamento, si sa, è d'ispirazione gramsciana. Ambedue i generi si collocano in rapporto polemico con il 'frammentismo', il quale giudicò banale e ripetitivo il romanzo ottocentesco. In una strana alleanza, fascisti e anti-fascisti riconoscono poi nei rispettivi generi il solido ed elementare riflesso di una nuova coscienza nazionale, nonché l'augurato allontanamento dai 'pezzettini' da 'letterati' (Vittorini).

I tre capitoli della seconda parte del volume sono interamente dedicati alla letteratura: successivamente al Rondismo, alla 'prosa d'arte' e alla nuova concezione calviniana del genere narrativo. Calligrafi e contenutisti si oppongono tra le due guerre. La pubblicazione di Rubè (1921) e de Gli Indifferenti (1929) spingono verso la difensiva i 'rondisti', sostenitori di una prosa lirica: Cecchi, Gargiulo, Falqui. Un intero capitolo, interessante e ben documentato, discute le numerose ambiguità sottostanti alle varie definizioni della 'prosa d'arte'. Di fronte al magma di nozioni proposte, sia da critici che da creatori, talvolta riuniti nella stessa persona, Pennings propone di ricorrere alla categoria trasversale del 'modo'. Vanta una lunga durata storica, è compatibile con più generi ed è meno legata a determinazioni formali. La proposta metodologica mi pare meritevole perchè permette di uscire dalla confusione del 'più o meno' del Falqui, dalla sovranità del fonismo protestata dal Gargiulo, dall'arbitrarietà categorica dei 'pesci rossi' del Cecchi. In altre parole: l'evolutività nozionale così riconosciuta permette di rendere conto di una pluralità di caratteristiche, inerente alla poetica. La stessa nozione di 'modo' viene riproposta nell'ultimo saggio dedicato al 'romanzesco' di Calvino. A Calvino non dispiace giocare nel bel mezzo di una 'piazza pulita', sgombra di generi letterari, preparata da Croce. La libertà ritrovata permette di esplorare l'intero paradigma del 'romanzesco', di portare lo sguardo oltre i modelli narrativi irrevocabili dell' Ottocento. Acquisiscono così diritto di cittadinanza romanzesca tanti libri 'aperti' (la lettera è del 1959) che definiranno il nuovo paradigma del romanzo novecentesco post-bellico. E in questo, non ultimo, prende posto il 'racconto' calviniano, declinato variamente, radicalmente esplorativo oltre i confini stabiliti, fino a toccare il territorio del 'poli-romanzo'. Le 'polemiche novecentesche' rivisitate dalla Pennings propongono, sotto la veste appunto 'polemica' del momento, numerose questioni di fondo per chi s'interessa al dibattito teorico delle arti e della letteratura in particolare.

\section{Walter Geerts}

Academia Belgica, Via Omero 8, I-00197 Roma, Italia. direttore@academiabelgica.it 